\title{
Doenças neurodegenerativas em adultos e idosos: um estudo epidemiológico descritivo
}

\author{
Neurodegenerative diseases in adults and the elderly: a \\ descriptive epidemiological study
}

\section{Enfermedades neurodegenerativas en adultos y ancianos: estudio epidemiológico descriptivo}

\author{
Erivânia Guedes da Paz ${ }^{1}$, Daiana de Jesus da Silva Mendes², Sabrina \\ Nogueira Brito ${ }^{3}$, Wilames Oliveira Barbosa 4
}

\begin{abstract}
1.Graduanda em Fisioterapia. Faculdade Metropolitana de Camaçari-Bahia, Brasil. https://orcid.org/00000001-7305-3961

2.Graduanda em Fisioterapia. Faculdade Metropolitana de Camaçari-Bahia, Brasil. https://orcid.org/0000$0001-9555-2518$

3.Fisioterapeuta. Residência em Saúde da Família (FESF/Fiocruz). Docente da Faculdade Metropolitana de Camaçari. Camaçari-Bahia, Brasil. https://orcid.org/0000-0001-8080-8488

4. Fisioterapeuta. Residência em Saúde do Adulto e do Idoso (HU/UFS), residência em Saúde da Família (FESF/Fiocruz). Docente da Faculdade Metropolitana de Camaçari. Camaçari-Bahia, Brasil. https://orcid.org/0000-0002-7526-6802
\end{abstract}

\section{Resumo}

Introdução. As doenças neurodegenerativas correspondem a moléstias causadas pela perda progressiva de neurônios nas estruturas do sistema nervoso, resultando em alterações funcionais gradativas. Objetivo. Descrever variáveis referentes as doenças neurodegenerativas em adultos e idosos no Brasil. Método. Trata-se de um estudo epidemiológico descritivo, construído a partir de dados coletados do Departamento de Informática do SUS, através da ferramenta TabNet. Foram incluídas, por meio da lista de morbidade CID-10, a doença de Alzheimer, doença de Parkinson e esclerose múltipla. Resultados. No período de dezembro de 2010 a dezembro de 2020, as internações por doenças neurodegenerativas totalizaram quase 51 mil casos, configurando aproximadamente 570 mil dias de permanência e pouco mais de 3 mil registros de óbitos. Conclusão. Verificouse uma tendência crescente nos registros de internações por doenças neurodegenerativas, bem como no número de óbitos. observa-se a necessidade de maiores investimentos em medidas que proporcionem um envelhecimento saudável.

Unitermos. Doenças Neurodegenerativas; Epidemiologia; Adulto; Idoso; Envelhecimento

\begin{abstract}
Introduction. Neurodegenerative diseases correspond to diseases caused by the progressive loss of neurons in the structures of the nervous system, resulting in gradual functional changes. Objective. describe variables related to neurodegenerative diseases in adults and the elderly in Brazil. Method. This is a descriptive epidemiological study, built from data collected from the SUS Department of Informatics, through the TabNet tool. The ICD-10 morbidity list included Alzheimer's disease, Parkinson's disease and multiple sclerosis. Results. In the period from december 2010 to december 2020, hospitalizations for neurodegenerative diseases totaled almost 51,000 cases, representing approximately 570,000 days of stay and just over 3,000 death records. Conclusion. There was a growing trend in the records of hospitalizations for neurodegenerative diseases, as well as in the number of deaths. there is a need for greater investments in measures that promote healthy aging.
\end{abstract}

Keywords. Neurodegenerative Diseases; Epidemiology; Adult; Aged; Aging 


\section{Resumen}

Introducción. Las enfermedades neurodegenerativas corresponden a la pérdida progresiva de neuronas en las estructuras del sistema nervioso, dando como resultado cambios funcionales graduales. Objetivo. Describir variables relacionadas con enfermedades neurodegenerativas en adultos y ancianos en Brasil. Método. Se trata de un estudio epidemiológico descriptivo, construido a partir de datos recolectados del Departamento de Informática del SUS, a través de la herramienta TabNet. A través de la lista de morbilidad de la CIE-10, se incluyeron la enfermedad de Alzheimer, la enfermedad de Parkinson y la esclerosis múltiple. Resultados. En el período de diciembre de 2010 a diciembre de 2020, las hospitalizaciones por enfermedades neurodegenerativas totalizaron casi 51.000 casos, lo que representa aproximadamente 570.000 días de estancia y algo más de 3.000 registros de defunción. Conclusión. Hubo una tendencia creciente en los registros de hospitalizaciones por enfermedades neurodegenerativas, así como en el número de defunciones. Es necesario realizar mayores inversiones en medidas que promuevan un envejecimiento saludable.

Palabras clave. Enfermedades neurodegenerativas; Epidemiología; Adulto; Anciano; Envejecimiento

Trabalho realizado na Faculdade Metropolitana de Camaçari-Bahia, Brasil.

Conflito de interesse: não

Recebido em: 26/06/2021

Aceito em: 23/08/2021

Endereço para correspondência: Wilames Oliveira Barbosa. Av. Rio Camaçari, Camaçari de Dentro. Camaçari-Bahia, Brasil. Email: will.trabalho@gmail.com

\section{INTRODUÇÃO}

As doenças neurodegenerativas correspondem a moléstias causadas pela perda progressiva de neurônios nas estruturas do sistema nervoso, resultando em alterações funcionais gradativas ${ }^{1}$. O desencadeamento de tais doenças pode ocorrer por diversos fatores como distúrbios genéticos, anormalidades proteicas, estresse oxidativo e exposição a substâncias tóxicas ${ }^{1-4}$. Caracterizam-se como sendo incuráveis, debilitantes, de início insidioso e progressão crônica ${ }^{5}$. Dentre estas, a doença de Alzheimer, a doença de Parkinson e a esclerose múltipla são as que mais acometem adultos maduros e idosos ${ }^{6-8}$.

As doenças neurodegenerativas apresentam altas taxas de morbidade e podem causar grandes perturbações corporais. Tais perturbações englobam tanto 
comprometimentos físicos quanto cognitivos, que posteriormente levam à morte do indivíduo. Em comum, tais doenças compartilham a perda de neurônios e a presença de proteínas mal enoveladas em áreas cerebrais ${ }^{5}$.

A crescente prevalência das doenças neurodegenerativas se deve, em parte, ao aumento da expectativa de vida, concomitante ao envelhecimento populacional ${ }^{6,9}$. O envelhecimento consiste em um processo natural caracterizado por diversas mudanças que ocorrem em todo o organismo, sendo o principal fator de risco para as doenças crônicas, o que culmina por gerar então uma estreita relação entre o avanço da idade e o surgimento de tais patologias ${ }^{10-12}$.

O Brasil vivencia o chamado período de transição demográfica, onde o número de idosos tende a crescer a ponto de seu aumento superar o número de jovens, que tende a cair. Estima-se que em 2060 mais de um terço da população brasileira será constituída de idosos, o que resultará em sobrecarga na saúde e aumento das doenças crônico-degenerativas ${ }^{13,14}$.

As mudanças no contexto demográfico que ocorrem em todo o mundo levam a consequentes alterações no perfil de saúde populacional. Considerando essas alterações e a complexidade dos distúrbios degenerativos, fazem-se necessários estudos que busquem investigar variáveis que envolvam tais doenças. Dessa forma, é possível sinalizar a necessidade de investimentos em saúde pública para que tais enfermidades possam ser prevenidas como também 
tratadas com todo aporte necessário, podendo o manejo da saúde envolver desde medicamentos à reabilitação dos pacientes. Neste sentido, o objetivo do estudo foi o de descrever variáveis referentes às doenças neurodegenerativas em adultos e idosos no Brasil.

\section{MÉTODO}

Trata-se de um estudo epidemiológico descritivo, realizado por meio de dados secundários coletados a partir do Departamento de Informática do Sistema Único de Saúde (DATASUS), por meio do Sistema de Informações Hospitalares do SUS (SIH/SUS), mediante a ferramenta TabNet. A abrangência geográfica envolveu o Brasil por região e unidade de federação.

A coleta abrangeu dados referentes ao período de dezembro de 2010 a dezembro de 2020. Através do capítulo VI do CID-10, foram incluídas doenças do sistema nervoso, e por meio da lista de morbidade, doença de Alzheimer, doença de Parkinson e esclerose múltipla foram selecionadas.

Foram coletadas as seguintes variáveis: número de internações hospitalares no Brasil, ano de atendimento, caráter de atendimento, região, dias de permanência, cor/raça, sexo, taxa de mortalidade, valor de serviços hospitalares, óbitos e faixa etária, considerando indivíduos a partir de 20 anos. Os dados foram coletados entre janeiro e fevereiro de 2021. 


\section{RESULTADOS}

No período de dezembro de 2010 a dezembro de 2020, as internações por doenças neurodegenerativas totalizaram quase 51 mil casos, configurando aproximadamente $570 \mathrm{mil}$ dias de permanência e pouco mais de 3 mil registros de óbitos. Tanto o número de internações quanto o de óbitos foi mais prevalente na região Sudeste com, respectivamente, $59,2 \%$ e $61,8 \%$, conforme mostram os Gráficos 1 e 2.

Gráfico 1. Internações por região 2010/2020. Fonte: Ministério de Saúde (SIH/SUS).

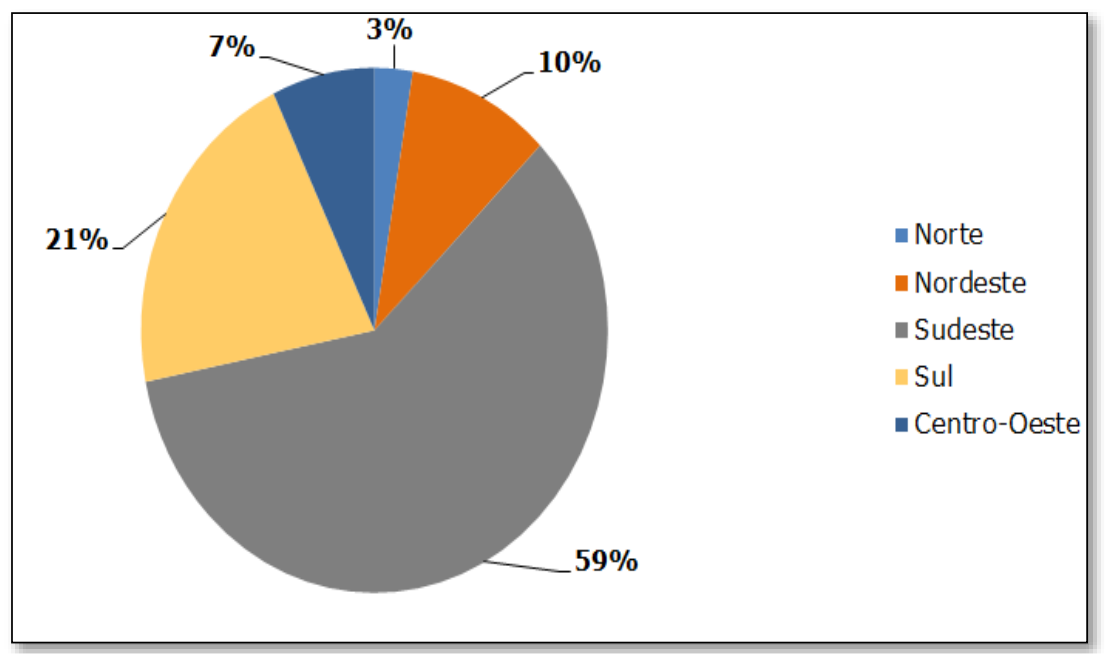

Gráfico 2. Óbitos por região 2010/2020. Fonte: Ministério de Saúde - (SIH/SUS).

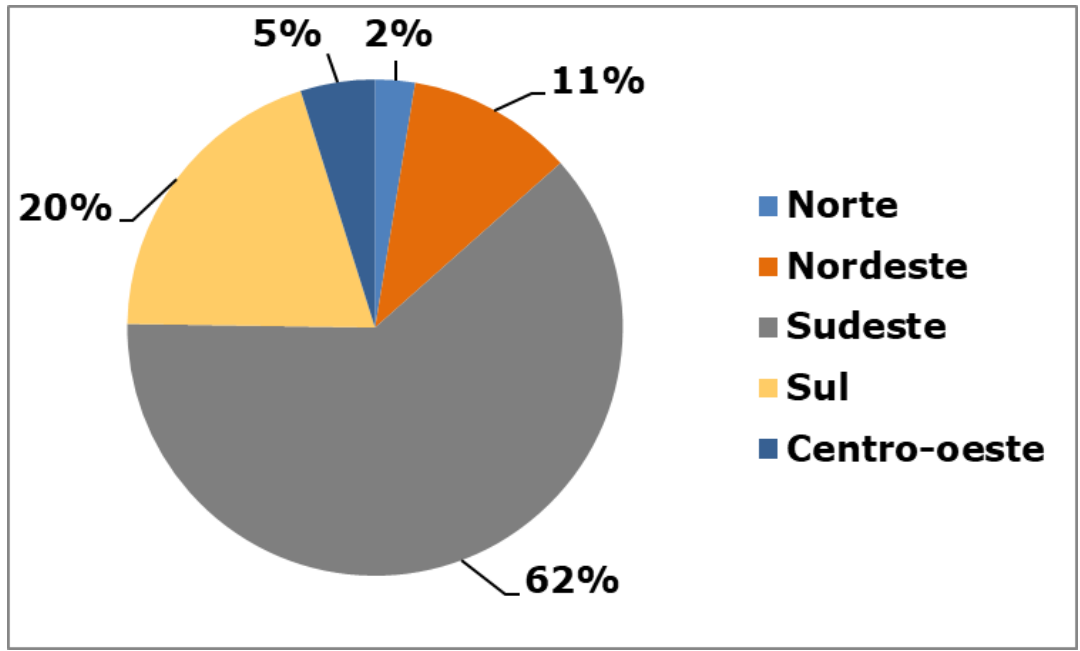


De acordo com a distribuição temporal, o ano de 2018 representou o maior número de hospitalizações, com 13,7\%, enquanto em 2019 ocorreu o maior registro de mortes, 14,8\%, conforme observado nos Gráficos 3 e 4.

Gráfico 3. Distribuição temporal por internações no Brasil 2010/2020. Fonte: Ministério de Saúde - (SIH/SUS).

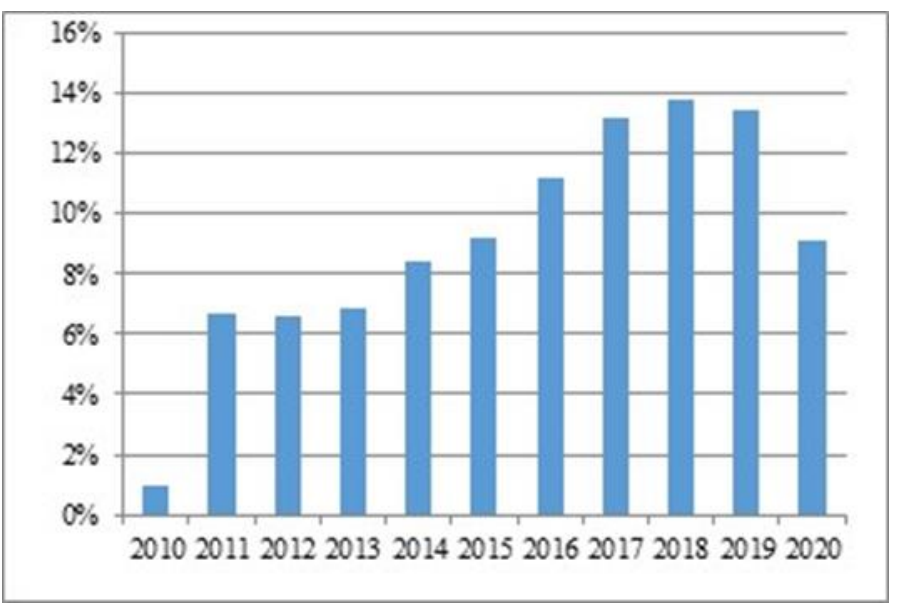

Gráfico 4. Distribuição temporal por óbitos no Brasil 2010/2020. Fonte: Ministério de Saúde - (SIH/SUS).

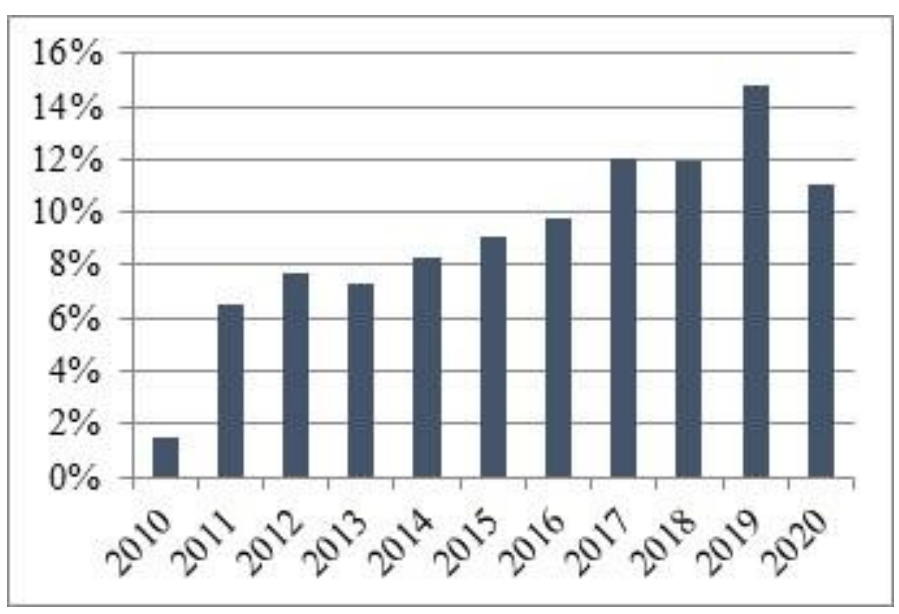

As internações foram mais frequentes nos indivíduos do sexo feminino, com $64 \%$ e na faixa etária a partir de 80 anos $(18,5 \%)$. Quanto ao grupo étnico, indivíduos de cor branca corresponderam aos maiores números de internações com $52 \%$. Em relação ao atendimento, 64,1\% ocorreram em 
caráter de urgência e 35,9\% foram por caráter eletivo (Tabela 1).

Tabela 1. Internações por região, sexo, raça, faixa etária e caráter de atendimento no Brasil entre dez/2010 e dez/2020.

\begin{tabular}{lc}
\hline Dados gerais & $\mathbf{n}(\mathbf{\%})$ \\
Sexo & \\
Feminino & \\
Masculino & $32.198(64,10)$ \\
Cor/raça & $18.385(35,90)$ \\
Branca & \\
Preta & \\
Parda & $26.327(52,05)$ \\
Amarela & $1.709(3,38)$ \\
Indígena & $10.598(20,95)$ \\
Sem informação & $405(0,80)$ \\
& $7(0,01)$ \\
Faixa - etária & $11.537(22,81)$ \\
& \\
20 a 29 & \\
30 a 39 & \\
40 a 49 & $7.010(13,86)$ \\
50 a 59 & $9.199(18,19)$ \\
60 a 69 & $7.315(14,46)$ \\
70 a 79 & $5.917(11,70)$ \\
80 e mais & $4.914(9,71)$ \\
Caráter de atendimento & $6.846(13,53)$ \\
Urgência & $9.352(18,53)$ \\
Eletivo & \\
\hline
\end{tabular}

Os distúrbios neurodegenerativos apresentaram uma taxa de mortalidade de $6,5 \%$ e um custo de aproximadamente 64 milhões em valores de serviços hospitalares.

\section{DISCUSSÃO}

Este estudo revelou uma quantidade relativamente pequena de hospitalizações por doenças 
neurodegenerativas, se comparada aos resultados obtidos em estudos que investigaram internações por outras patologias ${ }^{15}$. A menor quantidade de registros pode ser explicada pelo fato de os distúrbios neurológicos serem de diagnóstico complexo e envolverem a necessidade de recursos de alta complexidade ${ }^{16}$. Além disso, boa parte dos pacientes possuem acesso à reabilitação, contribuindo para a diminuição das internações ${ }^{17}$.

Os gastos relacionados às enfermidades neurológicas são bastante significativos, não apenas no âmbito hospitalar, mas também a nível ambulatorial e domiciliar, tendo em vista que são pacientes crônicos, com disfunções progressivas, por vezes com algum grau de dependência ${ }^{18}$.

Neste estudo foi possível verificar que os indivíduos com idades a partir de 80 anos apresentaram maior registro de internações. Condições como descontrole e agravo de doenças crônicas em idosos de idades mais avançadas resultam em maior necessidade de internações, especialmente em caráter de urgência ${ }^{19-21}$.

As hospitalizações ocorrem em sua maior parte no sexo feminino, podendo ser justificada pelo fato da composição feminina da população brasileira ser superior à população masculina e pela menor expectativa de vida dos homens, cerca de sete anos a menos ${ }^{22}$. Além disso, as mulheres procuram mais os serviços de saúde, viabilizando assim a identificação mais cedo de patologias, incluindo as neurodegenerativas ${ }^{23}$. 
Este estudo verificou que os registros das internações se mostraram maiores na região Sudeste e em indivíduos de cor branca, podendo ser explicado pelo fato de a região abrigar o maior número residentes, que corresponde a $44 \%$ da população brasileira24. Além disso, embora o Brasil contenha mais pardos, as diferenças históricas, sociais e étnico-raciais levam pessoas de cor branca a possuírem maior facilidade de acesso aos serviços de saúde 25,26.

\section{CONCLUSÕES}

Conforme os dados encontrados, verificou-se uma tendência crescente nos registros de internações por doenças neurodegenerativas, bem como no número de óbitos. As internações ocorreram, na maior parte, em caráter de urgência, podendo indicar agravos no quadro clínico dos pacientes. Além disso, as características das internações refletem a caracterização sociodemográfica da população brasileira, com uma maior proporção de mulheres, de residentes do Sudeste e pessoas de cor parda, refletindo nas condições de saúde dos indivíduos.

Deste modo, observa-se a necessidade de maiores investimentos em medidas que proporcionem um envelhecimento saudável. Ademais, é importante a realização de mais estudos que direcionem a identificação e tratamento precoce das doenças neurodegenerativas, visando uma melhor assistência a nível primário, reduzindo os números de internações e óbitos. 


\section{REFERÊNCIAS}

1.Marchi F, Contaldi E, Magistrelli L, Cantello R, Comi C, Mazzani L. Telehealth in Neurodegenerative Diseases: Opportunities and Challenges for Patients and Physicians. Brain Sci 2021;11:1-22. http://dx.doi.org/10.3390/brainsci11020237

2.Rekatsina M, Paladini A, Piroli A, Zis P, Pergolizzi JV, Varrassi G. Pathophysiology and Therapeutic Perspectives of Oxidative Stress and Neurodegenerative Diseases: A Narrative Review. Rev Adv Ther 2019;37:113-39. http://dx.doi.org/10.1007/s12325-019-01148-5

3.Sheikh S, Safia, Haque E, Mir SS. Neurodegenerative Diseases: Multifactorial Conformational Diseases and Their Therapeutic Interventions. J Neurodegener Dis 2013;2013:1-8. http://dx.doi.org/10.1155/2013/563481

4.Dugger BN, Dickson DW. Pathology of Neurodegenerative Diseases. Cold Spring Harb Perspect Biol 2017;9:1-22. http://dx.doi.org/10.1101/cshperspect.a028035

5. Love S. Post mortem sampling of the brain and other tissues in neurodegenerative disease. Histopathol 2004;44:304-17. http://dx.doi.org/10.1111/j.1365-2559.2004.01794.x

6. Gilter AD, Dhillon P, Shorter J. Neurodegenaritive diseases: models, mechanisms, and a new hope. Dis Models Mechan 2017;10:499-502. http://dx.doi.org/10.1242/dmm.030205

7.Schlachetzki J, Saliba SW, Oliveira ACP. Studying neurodegenerative diseases in culture models. Rev Bras Psiquiatr 2013;35:92-100. http://dx.doi.org/10.1590/1516-4446-2013-1159

8.Stephenson J, Nutma $E$, van der Valk $P$, Amor S. Inflammation in CNS neurodegenerative diseases. Immunology 2018;154:204-19. http://dx.doi.org/10.1111/imm.12922

9. Heemels MT. Neurodegenerative diseases. Nature 2016;539:179. http://dx.doi.org/10.1038/539179a

10. Hou Y, Dan X, Babbar M, Wei Y, Hasselbalch SG, Croteau DL, et al. Ageing as a risk factor for neurodegenerative disease. Nat Rev Neurol 2019;15:565-81. http://dx.doi.org/10.1038/s41582-019-0244-7

11.Cunnane SC, Trushina E, Morland C, Prigione A, Casadesus G, Andrews $\mathrm{ZB}$, et al. Brain energy rescue: an emerging therapeutic concept for neurodegenerative disorders of ageing. Nat Rev Drug Discov 2020;19:609-33. http://dx.doi.org/10.1038/s41573-020$\underline{0072-x}$

12.Beghi E. The Epidemiology of Epilepsy. Neuroepidemiology 2019;54:185-91. http://dx.doi.org/10.1159/000503831

13.Instituto Brasileiro de Geografia e Estatística. Síntese de indicadores sociais: uma análise das condições de vida da população brasileira (endereço na internet). Rio de Janeiro: Instituto Brasileiro de Geografia e Estatística; 2013 (acessado 02/2021).Disponível em: http://biblioteca.ibge.gov.br/visualizacao/livros/liv66777.pdf

14. Oliveira AS. Transição demográfica, transição epidemiológica e envelhecimento populacional no brasil. Rev Bras Geo Med Saúde 2019;15:69-79. http://dx.doi.org/10.14393/Hygeia153248614 
15.Boccolini CS. Morbimortalidade por doenças crônicas no Brasil: situação atual e futura. Rio de Janeiro: Fiocruz, 2016. https://saudeamanha.fiocruz.br/wpcontent/uploads/2017/11/PJSSaudeAmanha Texto0022 2016 v05.p df

16.Siuly S, Zhang Y. Medical big data: neurological diseases diagnosis through medical data analysis. Data Sci Eng 2016;1:54-64. http://dx.doi.org/10.1007/s41019-016-0011-3

17.Müller $A B$, Valentini NC, Pinto MEB. Fisioterapia em hospitalizações evitáveis por condições sensíveis à atenção primária. Fisioter Mov 2016;29:183-92.

http://dx.doi.org/10.1590/0103-

5150.029.001.AR03

18. Wynford-Thomas R, Robertson NP. The economic burden of chronic neurological disease. J Neurol 2017;264:2345-7. http://dx.doi.org/10.1007/s00415-017-8632-7

19.Rodrigues MM. Tendência das internações e da mortalidade de idosos por condições sensíveis à atenção primária. Rev Bras Epidemiol 2019;22:1-11. http://dx.doi.org/10.1590/1980-549720190010

20. Marques AP, Montilla DER, Almeida WS, Andrade CLT. Internação de idosos por condições sensíveis à atenção primária à saúde. Rev Saúde Pública 2014;48:817-26. http://dx.doi.org/10.1590/S00348910.2014048005133

21. Halter JB, Ouslander JG, Tinetti ME, Studenski S, High KP, Asthana S. Hazzard's Geriatric Medicine \& Gerontology. 6th ed. New York, McGraw Hill; 2009. https://accessmedicine. mhmedical.com/content.aspx?bookid=371\&se ctionid $=41587600$

22.Ginter $\mathrm{E}$, Simko V. Women live longer than men. Bratisl Lek Listy 2013;114:45-9. http://dx.doi.org/10.4149/bll 2013011

23. Teixeira DB. Atenção à saúde do homem: análise da sua resistência na procura dos serviços de saúde. Rev Cubana Enferm 2016;32. http://www.revenfermeria.sld.cu/index.php/enf/article/view/985/209 24.BNDS. Visconti GR, Santos MR. Região Sudeste: recuperando para desenvolver (acessado 02/2021). Disponível em: https://web.bndes.gov.br/bib/jspui/bitstream/1408/13074/3/Regi\%C $3 \%$ A30\%20Sudeste $\% 20-$

\%20recuperando\%20para\%20desenvolver 7 P BD.pdf

25. Araújo EM, Costa MCN, Hogan VK, Araújo TM, Dias AB, Oliveira LA. The use of the variable of race/color within Public Health: possibilities and limits. Interface 2009;13:383-94. http://dx.doi.org/10.1590/S1414-32832009000400012

26. Nelma NS, Favachol VBC, Boskall GA, Andrade EC, Merces NP, Oliveira MAF. Acesso da população negra a serviços de saúde: revisão integrativa. Rev Bras Enferm 2020;73:1-9. http://dx.doi.org/10.1590/0034-7167-2018-0834 